\title{
Supervisory Recurrent Fuzzy Neural Network Control of Wing Rock for Slender Delta Wings
}

\author{
Chih-Min Lin, Senior Member, IEEE, and Chun-Fei Hsu
}

\begin{abstract}
Wing rock is a highly nonlinear phenomenon in which an aircraft undergoes limit cycle roll oscillations at high angles of attack. In this paper, a supervisory recurrent fuzzy neural network control (SRFNNC) system is developed to control the wing rock system. This SRFNNC system is comprised of a recurrent fuzzy neural network (RFNN) controller and a supervisory controller. The RFNN controller is investigated to mimic an ideal controller and the supervisory controller is designed to compensate for the approximation error between the RFNN controller and the ideal controller. The RFNN is inherently a recurrent multilayered neural network for realizing fuzzy inference using dynamic fuzzy rules. Moreover, an on-line parameter training methodology, using the gradient descent method and the Lyapunov stability theorem, is proposed to increase the learning capability. Finally, a comparison between the sliding-mode control, the fuzzy sliding control and the proposed SRFNNC of a wing rock system is presented to illustrate the effectiveness of the SRFNNC system. Simulation results demonstrate that the proposed design method can achieve favorable control performance for the wing rock system without the knowledge of system dynamic functions.
\end{abstract}

Index Terms-Recurrent fuzzy neural network (RFNN), supervisory control, wing rock system.

\section{INTRODUCTION}

W HILE high-performance aircraft maneuver at high angles of attack, they may become unstable and enter into a limit cycle oscillation, mainly rolling motion known as wing rock [6], [7]. Because of the complex geometry of high-performance aircraft, it is difficult to isolate the various flow phenomena created by the forebody, strake and wing, or their relationship to the wing rock. Several theoretical and experimental studies have been proposed to determine or analyze the aeroelastic models from flight tests or wind-tunnel measurements [1], [4], [9], [15], [20], [21]. Recently, the control of the wing rock systems has become a significant research topic, and a series of papers considered the control based on output feedback linearization theory and adaptive control technique [13], [16], [17]. The neural-network-based control technique has represented an alternative design method for control of the dynamic systems to compensate the effects of nonlinearities and system uncertainties, so that the stability, convergence and robustness of the control system can be improved [5], [14], [18], [22], [25]. The

Manuscript received February 11, 2002; revised October 4, 2002 and November 26, 2003. This work was supported in part by the National Science Council of the Republic of China under Grant NSC 90-2213-E-155-016.

C.-M. Lin is with the Department of Electrical Engineering, Yuan-Ze University, Tao-Yuan 320, Taiwan, R.O.C. (e-mail: cml@ee.yzu.edu.tw).

C.-F. Hsu is with the Department of Electrical and Control Engineering, National Chiao-Tung University, Hsinchu 300, Taiwan, R.O.C. (e-mail:fei@cn.nctu.edu.tw).

Digital Object Identifier 10.1109/TFUZZ.2004.834803 fuzzy neural network (FNN) possesses advantages both of fuzzy systems and neural networks [2], [3], [11]. However, the neural networks presented in [2], [3], [5], [11], [14], [22], [25] are static feedforward networks. Recurrent neural networks have capabilities superior to the feedforward neural network, such as the dynamic response and the information storing ability [8], [10], [12]. Since the recurrent FNN captures the dynamic response of a system, the network model can be simplified.

In this paper, a supervisory recurrent fuzzy neural network control (SRFNNC) system is developed for the wing rock control system. This SRFNNC system is comprised of a recurrent fuzzy neural network (RFNN) controller and a supervisory controller. The RFNN controller is used to mimic an ideal controller and the supervisory controller is designed to compensate for the approximation error between the RFNN controller and the ideal controller. The RFNN is inherently a recurrent multilayered neural network with feedback connections in the second layer. An online parameter training methodology, using the gradient descent method and the Lyapunov stability theorem, is proposed to increase the learning capability. In addition, to relax the requirement for the uncertain bound in the supervisory controller, an estimation mechanism is incorporated to observe the uncertain bound. Thus, the chattering phenomena of the control efforts can be relaxed. Finally, a comparison between the sliding-mode control (SMC) [19], the fuzzy sliding control (FSC) [24] and the proposed SRFNNC is presented. Simulation results verify the effectiveness of the proposed SRFNNC system in achieving favorable control performance with unknown of system dynamic functions.

\section{Problem Statement and Mathematical Modeling}

The delta wing for the wing rock motion control is represented schematically in Fig. 1. This wing has one degree of freedom, and the dynamical system includes the wing (a flat uniform plate) and the parts of the string that rotate with it. The aerodynamic rolling moment is a complex nonlinear function of the rolling angle, roll rate, angle of attack and sideslip angle. The nonlinear wing rock equation of motion for an $80^{\circ}$ slender delta wing has been developed by Nayfeh et al. [15]. The differential equation describing the wing rock motion is given by [15], [18]

$$
\ddot{\phi}=\left(\frac{p U_{\infty}^{2} S b}{2 I_{x x}}\right) C_{l}+u
$$

where $\phi$ is the roll angle, an over-dot denotes a derivative with respect to time, $u$ is the control effort, $p$ is the density of air, $U_{\infty}$ is the freestream velocity, $S$ is the wing reference area, $b$ is 


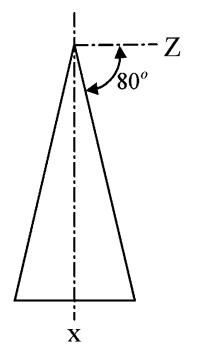

(a)

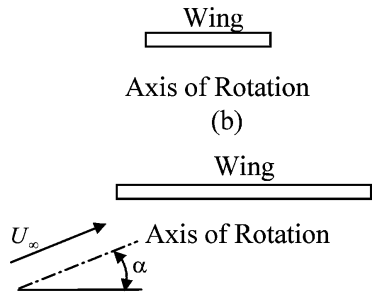

(c)

Fig. 1. Scheme of the delta wing: (a) plan view; (b) end view; (c) side view.

the chord, $I_{x x}$ is the mass moment of inertia, and $C_{l}$ is the roll moment coefficient. The roll-moment coefficient is written as

$$
C_{l}=c_{0}+c_{1} \phi+c_{2} \dot{\phi}+c_{3}|\phi| \dot{\phi}+c_{4}|\dot{\phi}| \dot{\phi}+c_{5} \phi^{3}
$$

The aerodynamic parameters $c_{i}$ are nonlinear functions of the angle of attack and have been derived in [15]. The numerical values of the parameters have been provided for different angles of attack for the $80^{\circ}$ slender delta wing. Substituting (2) into (1), system (1) can then be rewritten as

$$
\ddot{\phi}=f(\phi, \dot{\phi})+u
$$

where

$$
f(\phi, \dot{\phi})=b_{0}+b_{1} \phi+b_{2} \dot{\phi}+b_{3}|\phi| \dot{\phi}+b_{4}|\dot{\phi}| \dot{\phi}+b_{5} \phi^{3}
$$

and the parameters $b_{i}, i=0,1, \ldots, 5$ are given by

$$
b_{i}=\left(\frac{p U_{\infty}^{2} S b}{2 I_{x x}}\right) c_{i}
$$

The open-loop system time response with $u=0$ was simulated for two initial conditions: a small initial condition $(\phi=6 \mathrm{deg}$, $\left.\dot{\phi}=3 \mathrm{deg} \cdot \sec ^{-1}\right)$ and a large initial condition $(\phi=30 \mathrm{deg}$, $\left.\dot{\phi}=10 \mathrm{deg} \cdot \mathrm{sec}^{-1}\right)$. The phase-plane plot is shown in Fig. 2. For the small initial condition a limit cycle oscillation is obtained, and for the large initial condition the roll angle is divergent. Thus, it is shown that the uncontrolled nonlinear wing rock system will be unstable for some initial conditions.

Now, assuming that the aerodynamic parameters are known, the nominal model of the wing rock system can be represented as follows:

$$
\ddot{\phi}=f_{n}(\phi, \dot{\phi})+u
$$

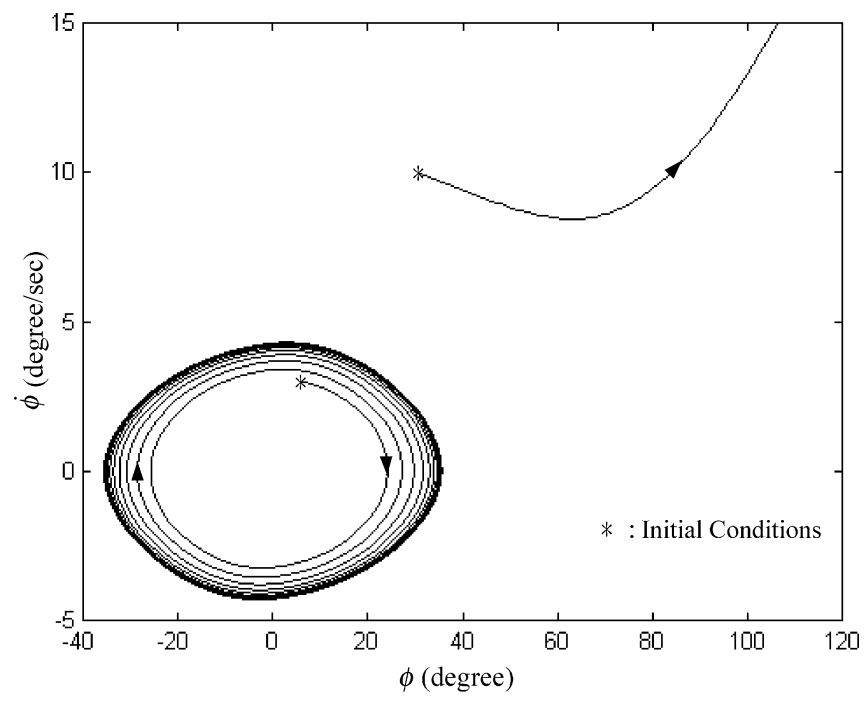

Fig. 2. Phase-plane portrait of uncontrolled wing rock motion system.

where $f_{n}(\phi, \dot{\phi})$ is the nominal value of $f(\phi, \dot{\phi})$. If uncertainties occur, i.e., the parameters of the system deviate from the nominal value, the controlled system can be formulated as

$$
\ddot{\phi}=f_{n}(\phi, \dot{\phi})+u+\Delta f(\phi, \dot{\phi})
$$

where $\Delta f(\phi, \dot{\phi})$ denotes the uncertainty with the assumption $|\Delta f(\phi, \dot{\phi})| \leq F$, in which $F$ is a positive constant.

\section{SMC}

A reference model is specified by a linear time-invariant differential equation

$$
\dot{\mathbf{x}}_{m}=\mathbf{A}_{m} \mathbf{x}_{m}
$$

where $\mathbf{x}_{m}=\left[\phi_{m}, \dot{\phi}_{m}\right]^{T}$ is the reference trajectory vector and

$$
\mathbf{A}_{m}=\left[\begin{array}{cc}
0 & 1 \\
-\omega_{n}^{2} & -2 \xi \omega_{n}
\end{array}\right]
$$

where $\xi>0$ is the damping ratio and $\omega_{n}>0$ is the natural frequency. For the choice of the damping ratio and natural frequency, $\mathbf{A}_{m}$ is a Hurwitz matrix and the reference trajectory vector $\mathbf{x}_{m}$ starting from any nonzero initial condition tends to zeros as $t \rightarrow \infty$. The control objective is to find a control law so that the roll angle $\phi$ can track the desired command $\phi_{m}$. Define the tracking error

$$
e=\phi_{m}-\phi
$$

Suppose that an integrated sliding function is defined as

$$
s=\dot{e}+k_{1} e+k_{2} \int_{0}^{t} e(\tau) d \tau
$$

where $k_{1}$ and $k_{2}$ are positive constants. The sliding-mode control law is defined as [19]

$$
u_{s m}=u_{e q}+u_{h t}
$$




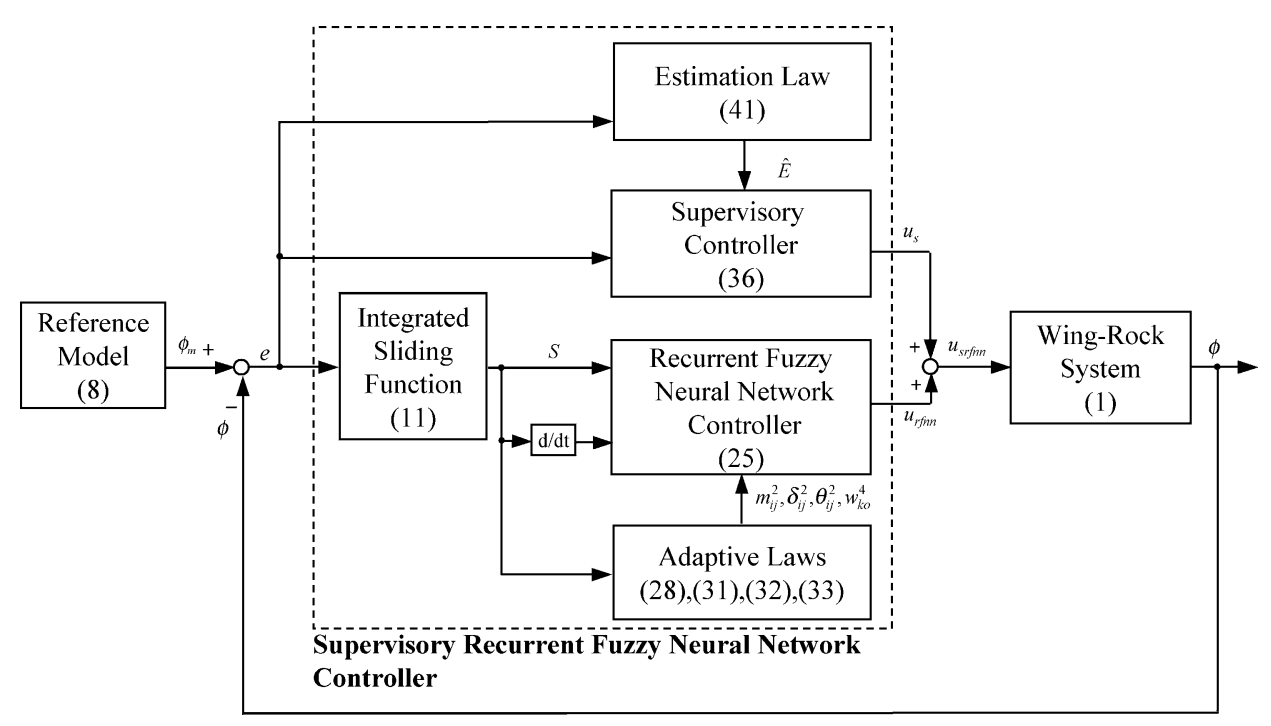

Fig. 3. Supervisory recurrent fuzzy neural network control wing rock motion system.

where the equivalent controller $u_{e q}$ is represented as

$$
u_{e q}=-f_{n}(\phi, \dot{\phi})+\ddot{\phi}_{m}+k_{1} \dot{e}+k_{2} e
$$

and the hitting controller $u_{h t}$ is designed to dispel the system uncertainties as

$$
u_{h t}=F \operatorname{sgn}(s)
$$

in which $\operatorname{sgn}(\cdot)$ is a sign function.

\section{SRFNN CONTROL}

If the system parameters are well known and measurable, an ideal controller can be obtained [19]

$$
u_{i d}=-f_{n}(\phi, \dot{\phi})-\Delta f(\phi, \dot{\phi})+\ddot{\phi}_{m}+k_{1} \dot{e}+k_{2} e .
$$

Substituting (15) into (7), gives

$$
\ddot{e}+k_{1} \dot{e}+k_{2} e=0 .
$$

If $k_{1}$ and $k_{2}$ are chosen to correspond to the coefficients of a Hurwitz polynomial, that is a polynomial whose roots lie strictly in the open left half of the complex plane, then $\lim _{t \rightarrow \infty} e=$ 0 . Since the system parameters may be unknown or perturbed, the ideal controller $u_{i d}$ is always unobtainable. Thus, an RFNN controller will be designed to approximate this ideal controller. In addition, a supervisory controller will be used to compensate for the approximation error between the RFNN controller and the ideal controller. The block diagram of the SRFNNC wing rock system is shown in Fig. 3, where the inputs of the RFNN controller are $s(t)$ and $\dot{s}(t)$. The SRFNNC is assumed to take the following form:

$$
u_{\mathrm{sffnn}}=u_{\mathrm{rfnn}}+u_{\mathrm{s}}
$$

where $u_{\mathrm{rfnn}}$ is to approximate the ideal controller in (15) and $u_{s}$ is the supervisory controller utilized to compensate the approximation error.

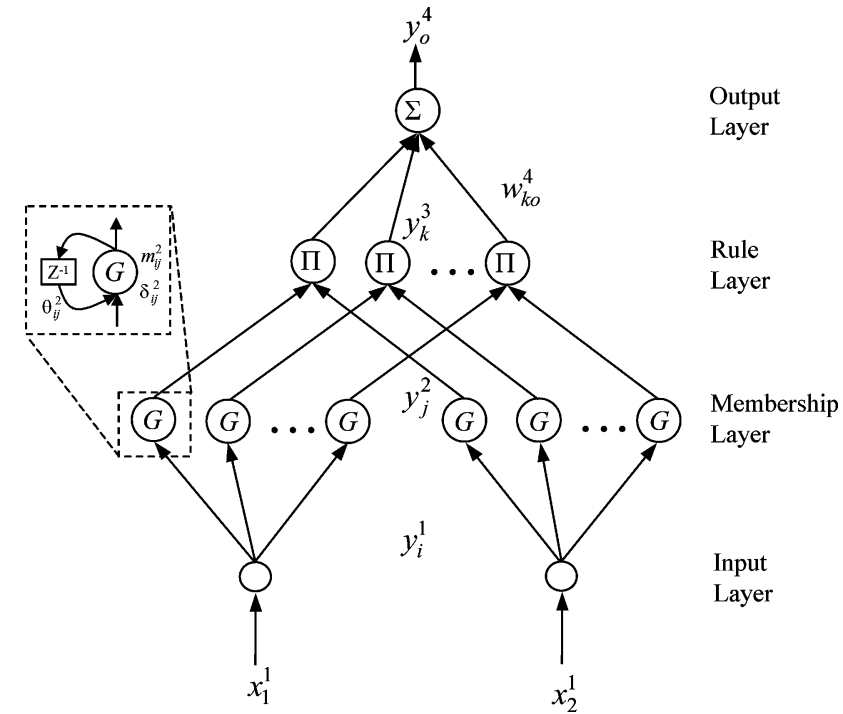

Fig. 4. Network structure of a recurrent RFNN.

\section{A. Recurrent Fuzzy Neural Network Controller}

A four-layer fuzzy neural network shown in Fig. 4, which is comprised of the input (the $i$ layer), membership (the $j$ layer), rule (the $\mathrm{k}$ layer), and output (the o layer) layers, is adopted to implement the proposed RFNN [10]. Layer 1 accepts the input variables. Layer 2 is used to calculate the Gaussian membership values. The nodes of layer 3 represent fuzzy rules. The links before layer 3 represent the preconditions of the rules, and the links after layer 3 represent the consequences of the rule nodes. Layer 4 is the output layer. The recurrent feedback is embedded in the network by adding feedback connections in the second layer of the fuzzy neural network. The signal propagation and the basic function in each layer are as follows.

- Layer 1-Input layer: For every node $i$ in this layer, the net input and the net output are represented as

$$
\begin{aligned}
\operatorname{net}_{i}^{1} & =x_{i}^{1} \\
y_{i}^{1} & =f_{i}^{1}\left(\operatorname{net}_{i}^{1}\right)=\operatorname{net}_{i}^{1}, \quad i=1,2
\end{aligned}
$$

where $x_{i}^{1}$ represents the $i$ th input to the node of layer 1 . 
- Layer 2-Membership layer: In this layer, each node performs a membership function and acts as a unit of memory. The Gaussian function is adopted as the membership function. For the $j$ th node

$$
\begin{aligned}
\operatorname{net}_{j}^{2} & =-\frac{\left(x_{i}^{2}+y_{j p}^{2} \theta_{i j}^{2}-m_{i j}^{2}\right)^{2}}{\left(\sigma_{i j}^{2}\right)^{2}} \\
y_{j}^{2} & =f_{j}^{2}\left(\operatorname{net}_{j}^{2}\right)=\exp \left(\operatorname{net}_{j}^{2}\right), \quad j=1,2, \ldots, m
\end{aligned}
$$

where $m_{i j}^{2}$ is the mean, $\sigma_{i j}^{2}$ is the standard deviation and $\theta_{i j}^{2}$ is the feedback gain of the Gaussian function in the $j$ th term of the $i$ th input linguistic variable $x_{i}^{2}$ to the node of layer 2, respectively, and $m$ is the total number of the linguistic variables with respect to the input nodes. It is clear that the feedback gain contains the memory terms $y_{j p}^{2}$, which denotes the output signal of layer 2 in the previous time.

- Layer 3-Rule layer: Each node $k$ in this layer is denoted by $\prod$, which multiplies the incoming signals and outputs the result of the product. For the $k$ th rule node

$$
\begin{aligned}
\operatorname{net}_{k}^{3} & =\prod_{j} w_{j k}^{3} x_{j}^{3} \\
y_{k}^{3} & =f_{k}^{3}\left(\operatorname{net}_{k}^{3}\right)=\operatorname{net}_{k}^{3}, \quad k=1,2, \ldots, n
\end{aligned}
$$

where $x_{j}^{3}$ represents the $j$ th input to the node of layer 3 , the weights $w_{j k}^{3}$ between the membership layer and the rule layer are assumed to be unity.

- Layer 4-Output layer: The single node $o$ in this layer is labeled as $\Sigma$, which computes the overall output as the summation of all incoming signals

$$
\begin{aligned}
\operatorname{net}_{o}^{4} & =\sum_{k} w_{k o}^{4} x_{k}^{4} \\
y_{o}^{4} & =f_{o}^{4}\left(\operatorname{net}_{o}^{4}\right)=\operatorname{net}_{o}^{4}, \quad o=1
\end{aligned}
$$

where the link weight $w_{k o}^{4}$ is the output action strength of the $o$ th output associated with the $k$ th rule, $x_{k}^{4}$ represents the $k$ th input to the node of layer 4 , and $y_{o}^{4}$ is the output of the recurrent FNN controller.

\section{B. Online Learning Algorithm}

In the SMC, the sliding condition is derived as $s \dot{s}<0$ such that the stability and convergence of $s \rightarrow 0$ as $t \rightarrow \infty$ can be guaranteed for the closed-loop system [19]. In order to train the RFNN, the online learning algorithm is a gradient descent algorithm in the space of network parameters and aims to minimize $s \dot{s}$ for achieving fast convergence of $s$. Therefore, $s \dot{s}$ is selected as the error function [11]. Taking the derivative of $s$ and using (3), it can be obtained that

$$
\dot{s}=\ddot{e}+k_{1} \dot{e}+k_{2} e=-f(\phi, \dot{\phi})-u+A_{d}\left(\phi_{m}, e\right)
$$

where $A_{d}\left(\phi_{m}, e\right)=\ddot{\phi}_{m}+k_{1} \dot{e}+k_{2} e$. Substituting (17) into (26), and multiplying both sides by $s$, gives

$$
s \dot{s}=-f(\phi, \dot{\phi}) s-\left(u_{\mathrm{rfnn}}+u_{s}\right) s+A_{d}\left(\phi_{m}, e\right) s .
$$

According to the gradient descent method, the weights in the output layer are updated by the following equation:

$$
\dot{w}_{k o}^{4}=-\eta_{w} \frac{\partial s \dot{s}}{\partial u_{\mathrm{srfnn}}} \frac{\partial u_{\mathrm{srfnn}}}{\partial w_{k o}^{4}}=-\eta_{w} \frac{\partial s \dot{s}}{\partial u_{\mathrm{rfnn}}} \frac{\partial u_{\mathrm{rfnn}}}{\partial w_{k o}^{4}}=\eta_{w} s x_{k}^{4}
$$

where the positive constant $\eta_{w}$ is a learning rate. Since the weight in the rule layer is a unity, only the approximation error term needs to be calculated and propagated by the following equation:

$$
\delta_{k}^{3} \equiv-\frac{\partial s \dot{s}}{\partial u_{\text {srfnn }}} \frac{\partial u_{\text {srfnn }}}{\partial \operatorname{net}_{o}^{4}} \frac{\partial \operatorname{net}_{o}^{4}}{\partial y_{k}^{3}} \frac{\partial y_{k}^{3}}{\partial \operatorname{net}_{k}^{3}}=s w_{k o}^{4} .
$$

The multiplication is done in the membership layer and the error term is computed as follows:

$$
\begin{aligned}
\delta_{j}^{2} & \equiv-\frac{\partial s \dot{s}}{\partial u_{\text {srfnn }}} \frac{\partial u_{\text {sfnn }}}{\partial \text { net }_{o}^{4}} \frac{\partial \text { net }_{o}^{4}}{\partial y_{k}^{3}} \frac{\partial y_{k}^{3}}{\partial \operatorname{net}_{k}^{3}} \frac{\partial \operatorname{net}_{k}^{3}}{\partial y_{j}^{2}} \frac{\partial y_{j}^{2}}{\partial \operatorname{net}_{j}^{2}} \\
& =\sum_{k} \delta_{k}^{3} y_{k}^{3} .
\end{aligned}
$$

The update laws of $m_{i j}^{2}, \sigma_{i j}^{2}$ and $\theta_{i j}^{2}$ can be obtained by the gradient search algorithm, i.e.,

$$
\begin{aligned}
\dot{m}_{i j}^{2} & \equiv-\eta_{m} \frac{\partial s \dot{s}}{\partial m_{i j}^{2}}=\eta_{m} \delta_{j}^{2} \frac{2\left(x_{i}^{2}+y_{j p}^{2} \theta_{i j}^{2}-m_{i j}^{2}\right)}{\left(\sigma_{i j}^{2}\right)^{2}} \\
\dot{\sigma}_{i j}^{2} & \equiv-\eta_{\sigma} \frac{\partial s \dot{s}}{\partial \sigma_{i j}^{2}}=\eta_{\sigma} \delta_{j}^{2} \frac{2\left(x_{i}^{2}+y_{j p}^{2} \theta_{i j}^{2}-m_{i j}^{2}\right)^{2}}{\left(\sigma_{i j}^{2}\right)^{3}} \\
\dot{\theta}_{i j}^{2} & \equiv-\eta_{\theta} \frac{\partial s \dot{s}}{\partial \theta_{i j}^{2}}=-\eta_{\theta} \delta_{j}^{2} \frac{2\left(x_{i}^{2}+y_{j p}^{2} \theta_{i j}^{2}-m_{i j}^{2}\right) y_{j p}^{2}}{\left(\sigma_{i j}^{2}\right)^{2}}
\end{aligned}
$$

where $\eta_{m}, \eta_{\sigma}$ and $\eta_{\theta}$ are the learning rates with positive constants.

\section{Supervisory Controller}

The most useful property of a neural network is its ability to approximate linear or nonlinear mapping through learning. By the universal approximation theorem [10], [23], there exists an optimal RFNN such that

$$
u_{i d}=u_{\mathrm{rfnn}}\left(\mathbf{w}^{*}\right)+\varepsilon
$$

where $\mathbf{w}^{*}=\left[\begin{array}{llll}w_{k o}^{4^{*}} & m_{i j}^{2^{*}} & \sigma_{i j}^{2^{*}} & \theta_{i j}^{2^{*}}\end{array}\right]^{T}$ is the ideal weight vector of the recurrent neural network controller, and $\varepsilon$ denotes the approximation error and is assumed to be bounded by $0 \leq$ 


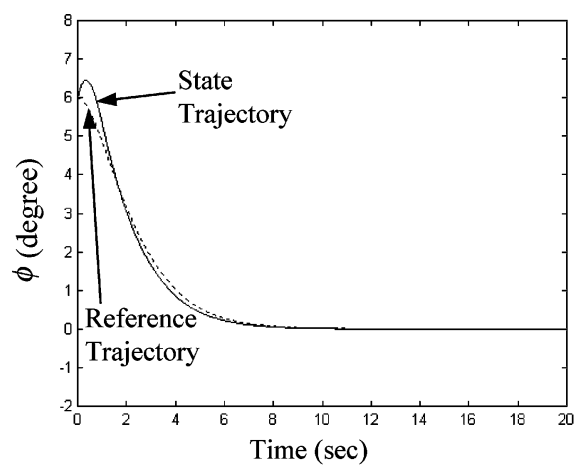

(a)

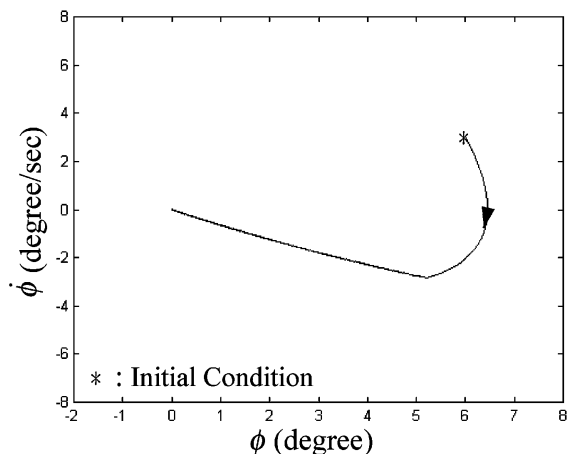

(b)

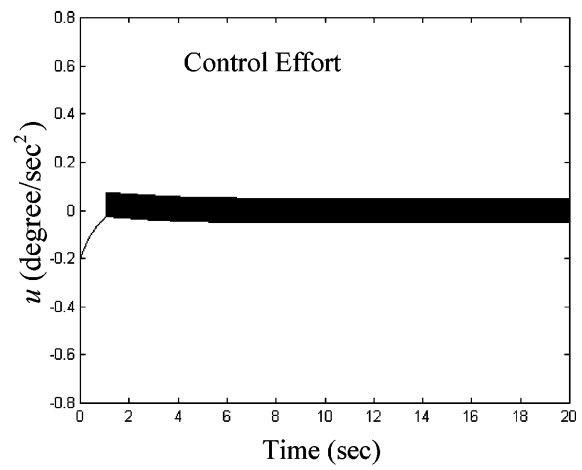

(c)

Fig. 5. Simulation results of SMC wing rock system for the small initial condition.

$|\varepsilon| \leq E$ where $E$ is a positive constant. The error bound is assumed to be a constant during the observation, however it is difficult to measure it in practical applications. Therefore, a bound estimation is developed to observe the bound of the approximation error. Define the estimation error of the bound

$$
\widetilde{E}=E-\hat{E}
$$

where $\hat{E}$ is the estimated error bound. The supervisory controller is designed to compensate for the effect of approximation error and is chosen as

$$
u_{s}=\hat{E} \operatorname{sgn}(s) .
$$

By substituting (17) into (7), it is revealed that

$$
\ddot{\phi}=f_{n}(\phi, \dot{\phi})+\left(u_{\mathrm{rfnn}}+u_{s}\right)+\Delta f(\phi, \dot{\phi}) \text {. }
$$

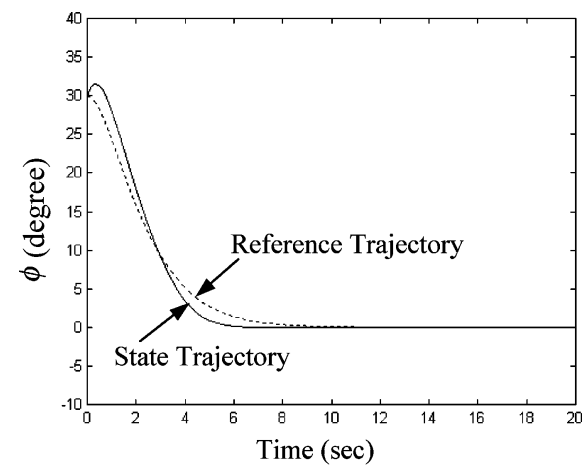

(a)

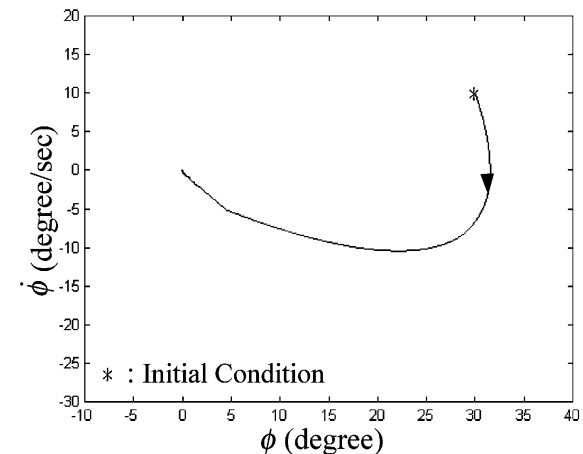

(b)

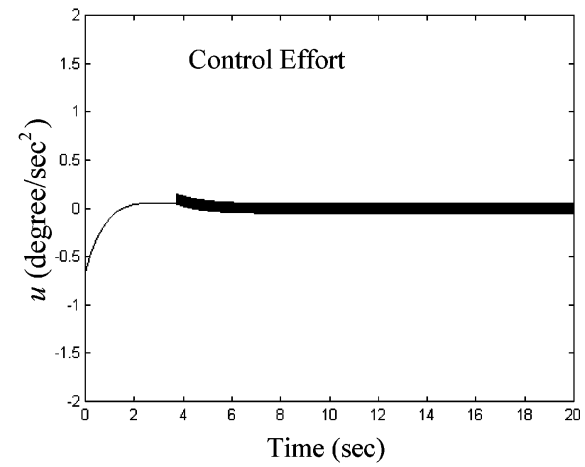

(c)

Fig. 6. Simulation results of SMC wing rock system for the large initial condition.

After some straightforward manipulation, the error equation governing the system can be obtained through (11), (15), and (34), as follows:

$$
\ddot{e}+k_{1} \dot{e}+k_{2} e=u_{i d}-u_{\mathrm{rfnn}}\left(\mathbf{w}^{*}\right)-u_{s}=\dot{s} .
$$

Define a Lyapunov function as

$$
V_{2}(s, \widetilde{E})=\frac{s^{2}}{2}+\frac{\widetilde{E}^{2}}{2 \eta_{E}}
$$

where the positive constant $\eta_{E}$ is a learning rate. Differentiating (39) with respect to time and using (34)-(36) and (38), it is obtained that

$$
\dot{V}_{2}(s, \widetilde{E})=s\left(\varepsilon-u_{s}\right)+\frac{\widetilde{E} \dot{\widetilde{E}}}{\eta_{E}}=\varepsilon s-\hat{E}|s|+\frac{\widetilde{E} \dot{\widetilde{E}}}{\eta_{E}} .
$$




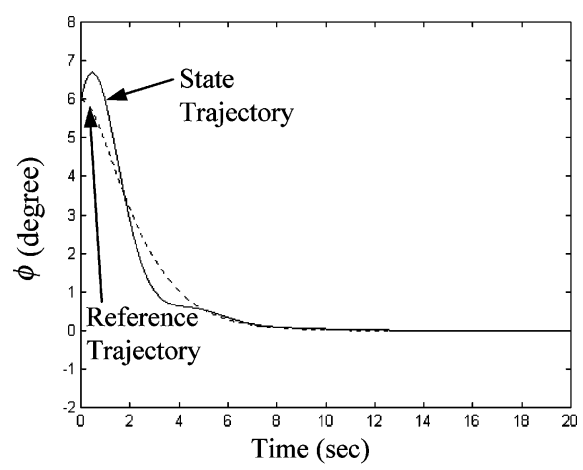

(a)

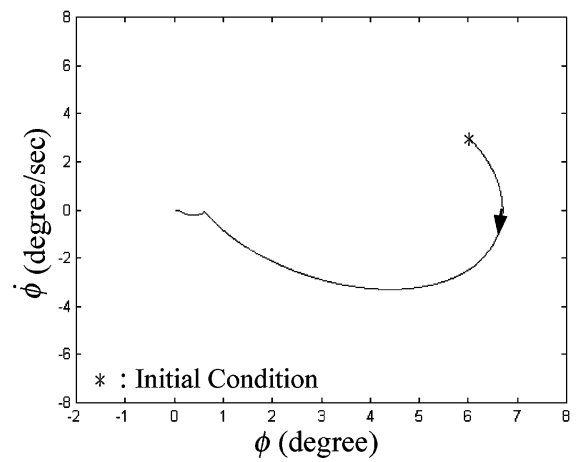

(b)

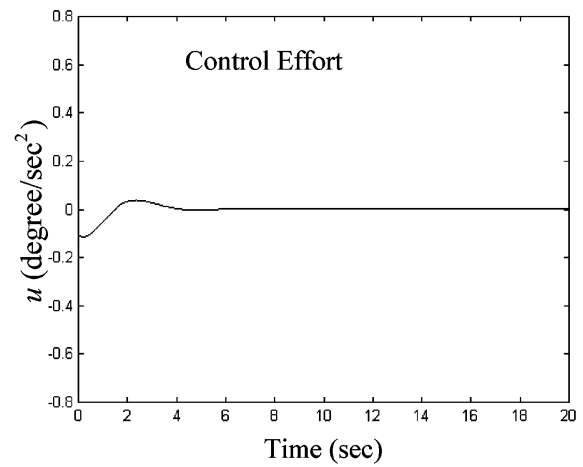

(c)

Fig. 7. Simulation results of FSC wing rock system for the small initial condition.

If the adaptive law of the supervisory controller is chosen as

$$
\dot{\widetilde{E}}=-\dot{\hat{E}}=-\eta_{E}|s|
$$

then (40) can be rewritten as

$$
\begin{aligned}
\dot{V}_{2}(s, \widetilde{E}) & =\varepsilon s-\hat{E}|s|-(E-\hat{E})|s| \\
& =\varepsilon s-E|s| \leq|\varepsilon||s|-E|s| \\
& =-(E-|\varepsilon|)|s| \leq 0 .
\end{aligned}
$$

Since $\dot{V}_{2}(s, \widetilde{E})$ is negative semi-definite, that is $V_{2}(s(t), \widetilde{E}(t)) \leq V(s(0), \widetilde{E}(0))$, it implies that $s$ and $\widetilde{E}$ are bounded. Let function $\Omega \equiv(E-|\varepsilon|)|s| \leq-\dot{V}_{2}(s, \widetilde{E})$, and integrate $\Omega(t)$ with respect to time, then it is obtained that

$$
\int_{0}^{t} \Omega(\tau) d \tau \leq V_{2}(s(0), \widetilde{E}(0))-V_{2}(s(t), \widetilde{E}(t)) .
$$

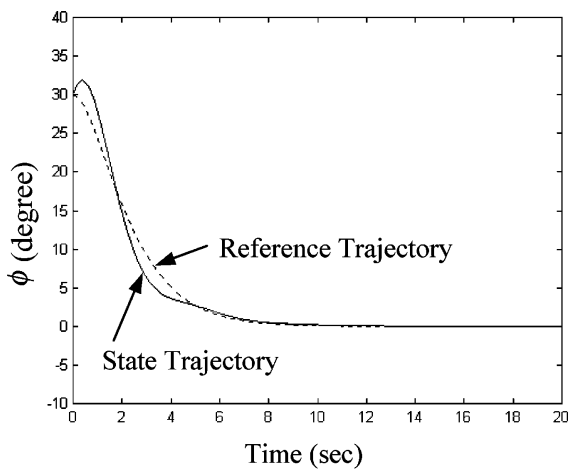

(a)

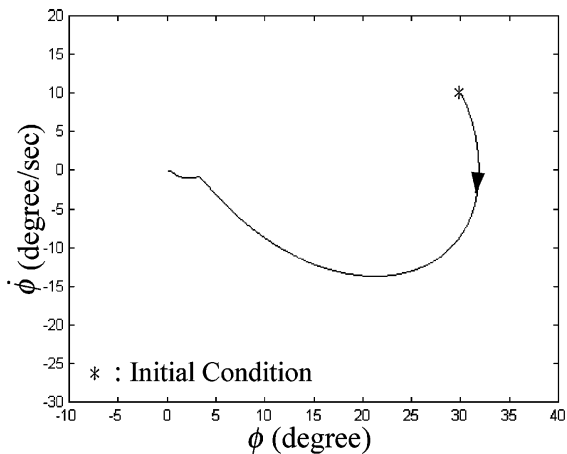

(b)

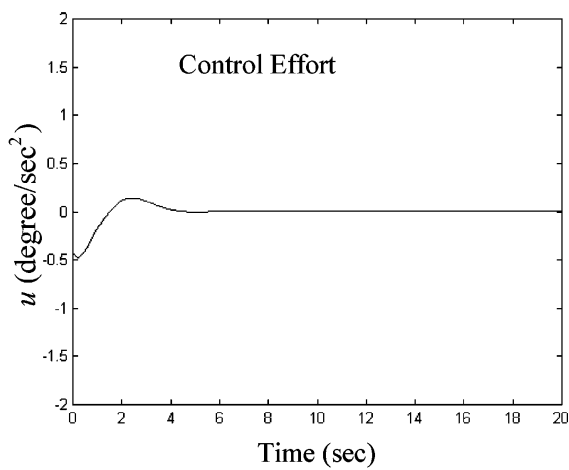

(c)

Fig. 8. Simulation results of FSC wing rock system for the large initial condition.

Because $V_{2}(s(0), \widetilde{E}(0))$ is bounded, and $V_{2}(s(t), \widetilde{E}(t))$ is nonincreasing and bounded, the following result can be obtained:

$$
\lim _{t \rightarrow \infty} \int_{0}^{t} \Omega(\tau) d \tau<\infty .
$$

Also, $\dot{\Omega}(t)$ is bounded, so by Barbalat's Lemma [19], $\lim _{t \rightarrow \infty} \Omega=0$. That is, $s \rightarrow 0$ as $t \rightarrow \infty$. Hence, the supervisory recurrent fuzzy neural network control system is asymptotically stable.

\section{Simulation Results}

The aerodynamic parameters of the delta wing for $25^{\circ}$ angle of attack are used for simulation. It is assumed that $U_{\infty}=$ $15 \mathrm{~m} / \mathrm{s}$ and $b=0.429 \mathrm{~m}$. The parameter $b_{i}$ for the model (3) are given by [15], [18]

$$
\begin{aligned}
& b_{0}=0, b_{1}=-0.01859521, b_{2}=0.015162375, \\
& b_{3}=-0.06245153, b_{4}=0.00954708, b_{5}=0.02145291 .
\end{aligned}
$$




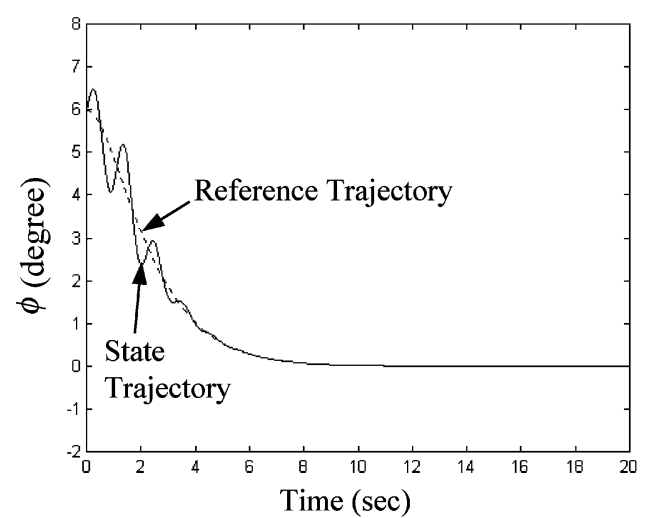

(a)

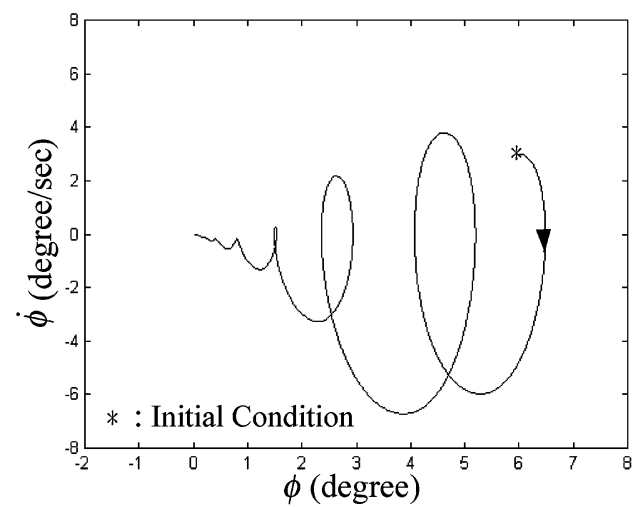

(b)

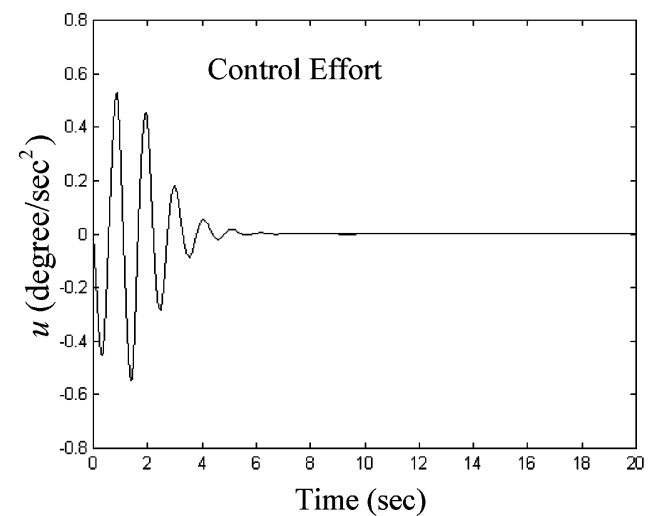

(c)
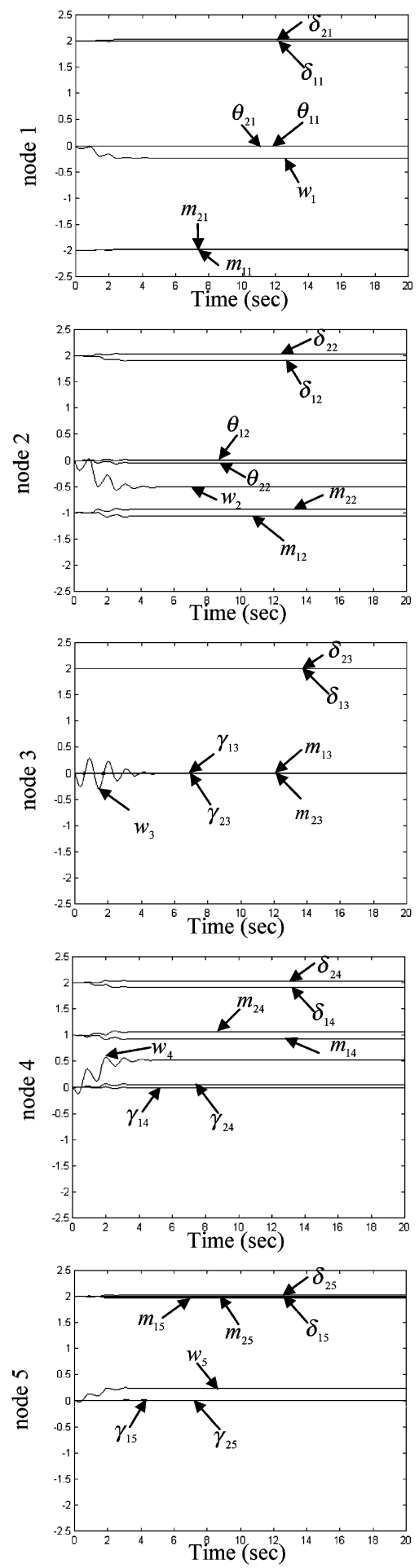

(d)

Fig. 9. Simulation results of SRFNNC wing rock system for the small initial condition.

The reference trajectory vector is chosen for $\mathbf{x}_{m} \rightarrow 0$ as $t \rightarrow \infty$ with the parameters $\xi$ and $\omega_{n}$ chosen as 1 and 0.8 , respectively. It should be emphasized that the derivation of SRFNNC does not need to use the aerodynamic parameters and the structure of the aerodynamic functions. The system parameters are used only for simulations. For practical implementation, the SRFNNC parameters can be tuned online by the proposed adaptive laws without the need of the system parameters. A RFNN with five hidden layer neurons in the rule layer is utilized to approximate the ideal controller. To investigate the effectiveness of the developed control system, two initial conditions (small initial condition $\phi(0)=6 \mathrm{deg}, \dot{\phi}(0)=3 \mathrm{deg} \cdot \mathrm{sec}^{-1}$ and large initial condition $\phi(0)=30 \mathrm{deg}, \dot{\phi}(0)=10 \mathrm{deg} \cdot \sec ^{-1}$ ) are used for simulation. For a stable system, the coefficients in (16) are chosen as $k_{1}=1.6$ and $k_{2}=0.64$. The sampling time is $0.002 \mathrm{~s}$. The parameters of the proposed SRFNNC system are selected as follows:

$$
\eta_{w}=\eta_{m}=\eta_{\sigma}=\eta_{\theta}=20 \text { and } \eta_{E}=0.1 .
$$




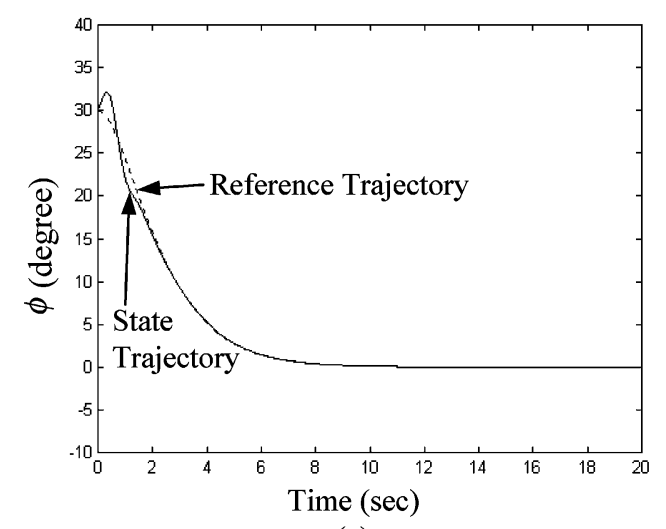

(a)

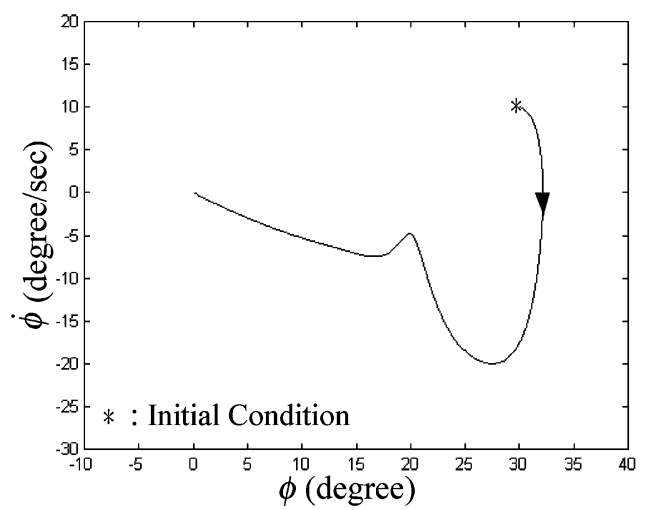

(b)

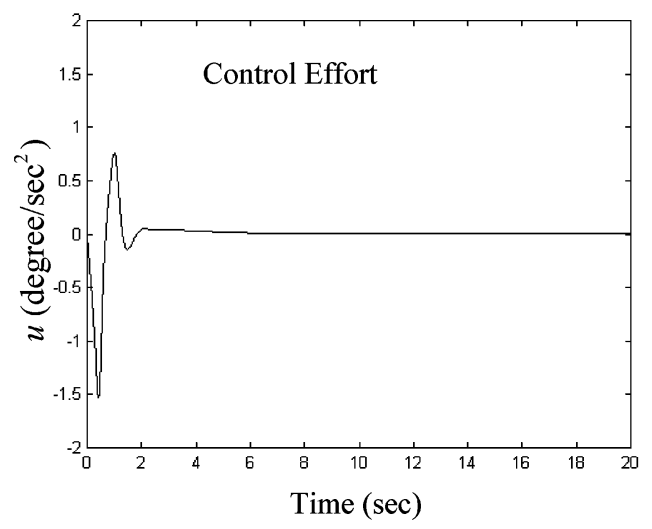

(c)
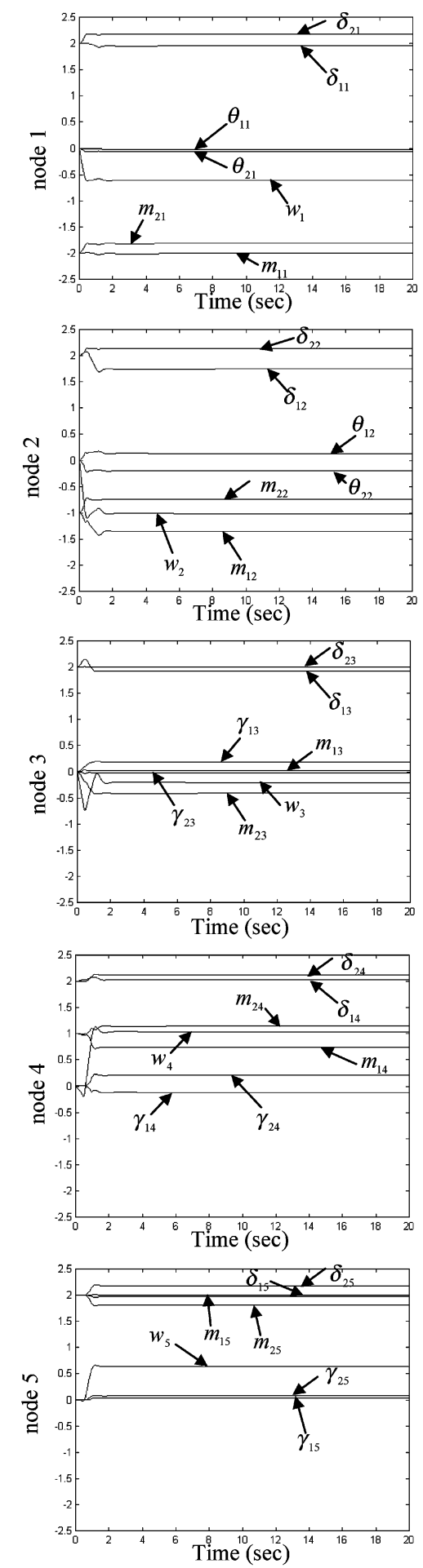

(d)

Fig. 10. Simulation results of SRFNNC wing rock system for the large initial condition.

Firstly, the SMC in [19] is applied to control the wing rock system. The simulation results of the SMC for small and large initial conditions with uncertainty bound $F=0.05$ are shown in Figs. 5 and 6, respectively. The state responses are shown in Figs. 5(a) and 6(a), the phase-plane portraits are shown in Figs. 5(b) and 6(b), and the associated control efforts are shown in Figs. 5(c) and 6(c), respectively. Simulation results show that the robust tracking performance has been achieved for different initial conditions. However, the chattering phenomena of the control efforts shown in Figs. 5(c) and 6(c) are undesirable. Second, the FSC in [24] is applied to control the wing rock system. The simulation results of the FSC for small and large initial conditions are shown in Figs. 7 and 8, respectively. These results show that not only the tracking performance can be achieved but also the control chattering can be eliminated. Finally, the developed SRFNNC is applied to control the wing rock system for comparing. The simulation results of the SRFNNC for small and large initial conditions are shown in 


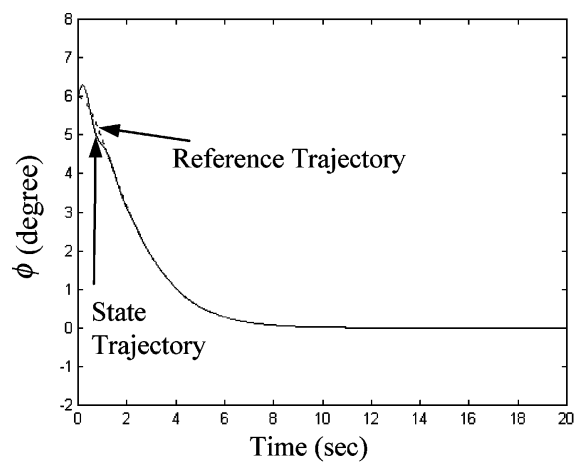

(a)

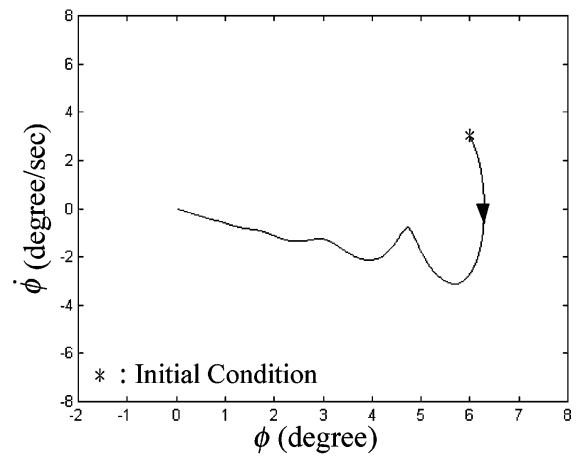

(b)

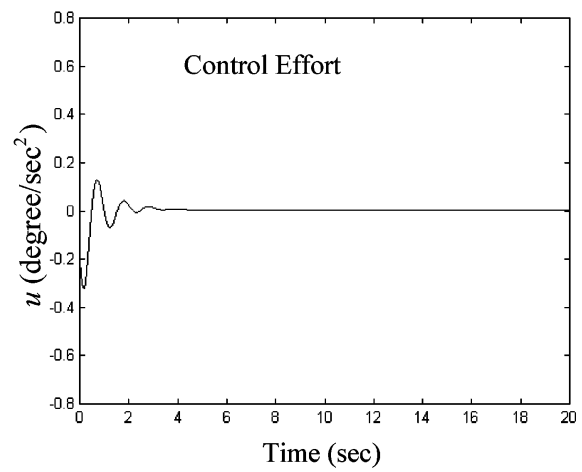

(c)

Fig. 11. Simulation results of trained SRFNNC wing rock system for the small initial condition.

Figs. 9 and 10, respectively. From these simulation results, it can be seen that robust tracking performance can be also achieved; and moreover, the chattering phenomena is much reduced in the control effort due to the on-line adaptation of the bound value in the supervisory controller. However, since the control rules are initialized from zero, the SRFNNC has the drawback of large transient responses of the state trajectories and control efforts at the initial learning phase. After $20 \mathrm{~s}$ of training in these simulations, the trained SRFNNC is applied to control the wing rock system again. The simulation results of this trained SRFNNC for small and large initial conditions are shown in Figs. 11 and 12, respectively. From these simulation results, it shows that the tracking performance of the trained SRFNNC is better than SMC and FSC.

\section{CONCLUSION}

In this paper, an SRFNNC system is developed for a wing rock system with significant uncertainties on the system dynam-

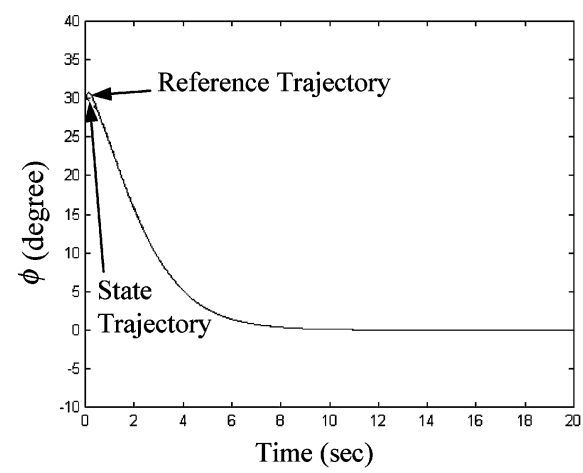

(a)

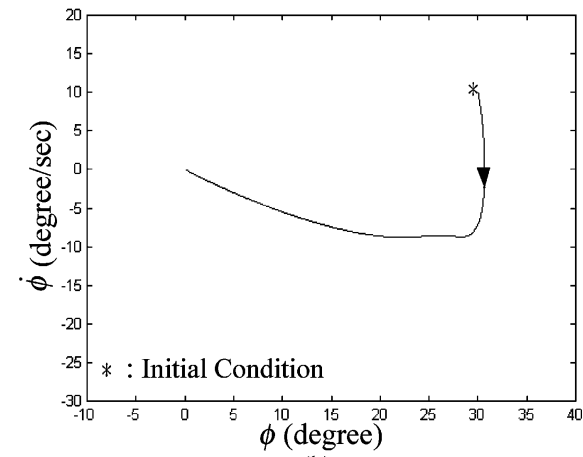

(b)

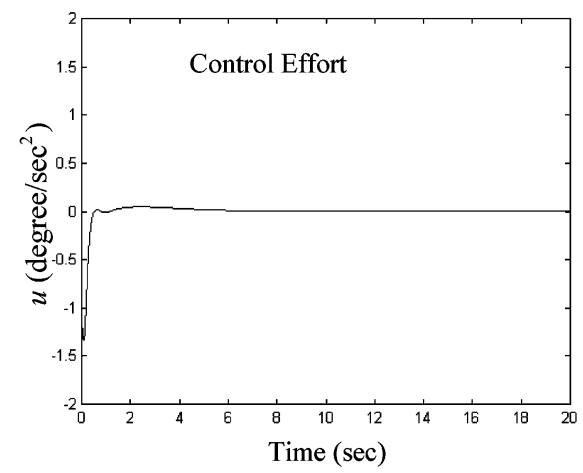

(c)

Fig. 12. Simulation results of trained SRFNNC wing rock system for the large initial condition.

ical behaviors. The SRFNNC system is comprised of an RFNN controller and a supervisory controller. The RFNN controller is investigated to mimic an ideal controller and the supervisory controller is designed to compensate for the approximation error between the RFNN controller and the ideal controller. For comparison, an SMC, a fuzzy sliding control and the proposed SRFNNC are simulated for the wing rock system. Simulation results demonstrate that the trained SRFNNC can achieve the best control performance for wing rock control.

\section{ACKNOWLEDGMENT}

The authors would like to thank the reviewers for their valuable comments.

\section{REFERENCES}

[1] S. Andrew, J. Arena, and C. N. Robert, "Experimental investigations on limit cycle wing rock of slender wings," J. Aircraft, vol. 31, pp. $1148-1155,1994$. 
[2] C. T. Chao and C. C. Teng, "Implementation of fuzzy inference systems using a normalized fuzzy neural network," Fuzzy Sets Syst., vol. 75, pp. 17-31, 1995.

[3] Y. C. Chen and C. C. Teng, "A model reference control structure using a fuzzy neural network," Fuzzy Sets Syst., vol. 73, pp. 291-312, 1995

[4] L. E. Ericsson, "Wing rock analysis of slender delta wings, review and extension," J. Aircraft, vol. 32, pp. 1221-1226, 1995.

[5] S. S. Ge, C. C. Hang, and T. Zhang, "Adaptive neural network control of nonlinear systems by state and output feedback," IEEE Trans. Syst., Man, Cybern. B, vol. 29, pp. 818-828, Oct. 1999.

[6] C. Hsu and C. E. Lan, "Theory of wing rock," J. Aircraft, vol. 22, pp. 920-924, 1985

[7] P. Konstadinopoulos, D. T. Mook, and A. H. Nayfeh, "Subsonic wing rock of slender delta wings," J. Aircraft, vol. 22, pp. 223-228, 1985.

[8] C. C. $\mathrm{Ku}$ and K. Y. Lee, "Diagonal recurrent neural networks for dynamic systems control," IEEE Trans. Neural Networks, vol. 6, pp. 144-156, Feb. 1995.

[9] C. E. Lan, Y. Chen, and K. J. Lin, "Experimental and analytical investigations of transonic limit-cycle oscillations of a flaperon," J. Aircraft, vol. 32, pp. 905-910, 1995.

[10] C. H. Lee and C. C. Teng, "Indentification and control of dynamic systems using recurrent fuzzy neural networks," IEEE Trans. Fuzzy Syst., vol. 8, pp. 349-366, Apr. 2000.

[11] C. M. Lin and C. F. Hsu, "Neural-network-based adaptive control for induction servomotor drive systems," IEEE Trans. Ind. Electron., vol. 49, pp. 115-5123, Jan. 2002.

[12] F. J. Lin and R. J. Wai, "Hybrid control using recurrent fuzzy neural network for linear-induction motor servo drive," IEEE Trans. Fuzzy Syst., vol. 9, pp. 102-115, Feb. 2001.

[13] M. M. Monahemi and M. Krstic, "Control of wing rock motion using adaptive feedback linearization," J. Guid., Control, Dyna., vol. 19, pp. 905-912, 1996.

[14] K. Nam, "Stabilization of feedback linearizable systems using radial basis function network," IEEE Trans. Automat. Contr., vol. 44, pp. 1026-1031, June 1999.

[15] A. H. Nayfeh, J. M. Elzebda, and D. T. Mook, "Analytical study of the subsonic wing-rock phenomenon for slender delta wings," J. Aircraft, vol. 26, pp. 805-809, 1989.

[16] S. P. Shue, M. E. Sawan, and K. Rokhsaz, "Optimal feedback control of a nonlinear system: Wing rock example," J. Guid., Control, Dyna., vol. 19, pp. 166-171, 1996.

[17] S. P. Shue and R. K. Agarwal, "Nonlinear $H_{\infty}$ method for control of wing rock motions," J. Guid., Control, Dyna., vol. 23, pp. 60-68, 2000.

[18] S. N. Singh, W. Yim, and W. R. Wells, "Direct adaptive and neural control of wing-rock motion of slender delta wings," J. Guid., Control, Dyna., vol. 18, pp. 25-30, 1995.

[19] J. J. E. Slotine and W. P. Li, Applied Nonlinear Control. Upper Saddle River, NJ: Prentice-Hall, 1991.

[20] S. Y. Tan and C. E. Lan, "Estimation of aeroelastic models in structural limit-cycle oscillations from test data," J. AIAA, vol. 35, pp. 1025-1029, 1997.

[21] T. L. Trankle and S. D. Bachner, "Identification of a nonlinear aerodynamic model of the F-14 aircraft," J. Guid., Control, Dyna., vol. 18, pp. 1292-1297, 1995.
[22] H. Wang and Y. Wang, "Neural-network-based fault-tolerant control of unknown nonlinear systems," Proc. Inst. Elect. Eng.Control Theory Applications, vol. 146, pp. 389-398, 1999.

[23] L. X. Wang, Adaptive Fuzzy Systems and Control: Design and Stability Analysis. Upper Saddle River, NJ: Prentice-Hall, 1994.

[24] L. K. Wong, F. H. F. Leung, and P. K. S. Tam, "A fuzzy sliding controller for nonlinear systems," IEEE Trans. Ind. Electron., vol. 48, pp. 32-37, Jan. 2001

[25] M. Zhihong, H. R. Wu, and M. Palaniswami, "An adaptive tracking controller using neural networks for a class of nonlinear systems," IEEE Trans. Neural Networks, vol. 9, pp. 947-1031, Dec. 1998.

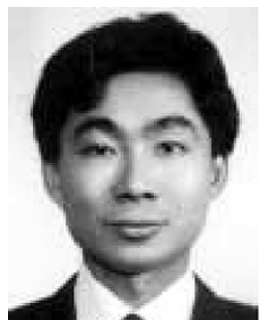

Chih-Min Lin (S'86-M'87-S'99) received the B.S. and M.S. degrees in control engineering and the Ph.D. degree in electronics engineering from National Chiao Tung University, Taiwan, R.O.C., in 1981, 1983, and 1986, respectively.

During 1986-1992, he was with the Chung Shan Institute of Science and Technology, Taiwan, as a Deputy Director of system engineering of missile systems. He also served concurrently as an Associate Professor at Chiao Tung University and Chung Yuan University, Taiwan. He joined the Faculty of the Department of Electrical Engineering, Yuan-Ze University, Taiwan, in 1993, and is currently a Professor and the Chairman of the Department of Electrical Engineering. During 1997-1998, he was an Honorary Research Fellow at the University of Auckland, Auckland, New Zealand. His research interests include fuzzy neural network, cerebellar model articulation control, guidance and flight control, and systems engineering.

Dr. Lin has also served as a Committee Member of the Chinese Automatic Control Society and the Deputy Chairman of the IEEE Control Systems Society, Taipei Section.

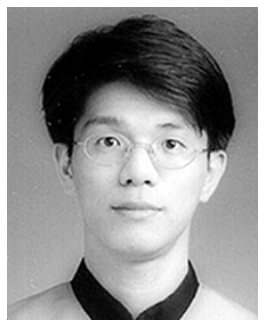

Chun-Fei Hsu received the B.S., M.S., and Ph.D. degrees in electrical engineering from Yuan-Ze University, Taiwan, R.O.C., in 1997, 1999 and 2002, respectively.

After graduation, he joined the Department of Electrical and Control Engineering, National Chiao-Tung University, Taiwan. During 2002-2003, he was doing postdoctoral research on virtual-reality dynamic simulators and intelligent transportation systems. His research interests include servomotor drives, adaptive control, and flight control and intelligent control using fuzzy system and neural network technologies. 